\title{
On Katz and Indeterminacy of Translation
}

\author{
NANCY S. BRAHM \\ University of Nebraska
}

In Word and Object, Quine sets forth and defends the thesis of the indeterminacy of translation. 1 The indeterminacy thesis is designed to reveal the empirical emptiness of the pre-critical notion of meaning. Quine also believes himself to have exposed (though, strictly speaking, not argued for) the shortcomings of the pre-critical notion of meaning ${ }^{2}$ by subjecting it to the empirical, behavioristic spotlight of radical translation. If Quine is correct, if radical translation is impossible, then the indeterminacy thesis must therefore be accepted as a consequence. Moreover, once such indeterminacy and the consequences which accompany it are fully appreciated, our widespread, common-sense notions of meaning and sameness of meaning must be discarded.

Quine's indeterminacy thesis has been the target of much criticism. ${ }^{3}$ Yet many of these criticism are the result of misunderstanding (or perhaps simply missing the point of Quine's work. Jerrold Katz is one such critic who claims to refute Quine's thesis. In his recent article, "The Refutation of Indeterminacy," Katz ventures to say that if he is correct, "there is a straightforward sense in which the indeterminacy thesis is refuted."4

I will attempt to show that Katz is not correct, the refutation he proposes is neither straightforward nor a successful refutation of Quine's indeterminacy thesis. Thus, I contend that Quine's indeterminacy thesis can easily withstand Katz's alleged refutation. This paper will consist of two main sections, the first of which will outline Quine's indeterminacy thesis and provide concomitant arguments, while the second section will evaluate Katz's proposed refutation of the indeterminacy thesis. The former section is intended to serve as a basis for evaluating Katz's charges against Quine. In the latter section 1 will point out precisely where and why Katz's alleged refutation fails.

1 W.V.W. Quine, Word and Object (Cambridge, Mass.: MIT Press, 1960).

2 The traditional, or precritical notion of meaning to which I refer is one of fixed, unique meanings to which particular thoughts or sentences are believed to correspond. In short, these meanings are intensional entities which are thought to get captured (represented, expressed, etc.) by sentences: or terms.

3 For example, see D. Davidson and J. Hintikka (eds.), Words and Objeclions: Essinys on the Work of W. V. Quine (Dordrecht: Reidel, 1968).

4 J. J. Katz, "The Refutation of Indeterminacy," The Journal of Philosophy, LXXXV, 5 (May 1988): 228. 


\section{The Indeterminacy Thesis and Supporting Arguments5}

In Word and Object we find the early formulation of the indeterminacy thesis, as well as the famous experiment of radical translation. There Quine first argues for the thesis, 6 although as Quine himself will later remark, this argument proves to be neither the strongest nor the preferred argument. It is in later writings, namely, "On the Reasons for Indeterminacy of Translation," "Indeterminacy of Translation Again," "Epistemology Naturalized," as well as responses provide to essays in Words and Objections, that we find different, refined (and often clearer) argumentation for the indeterminacy thesis. 7 We will now consider that early formulation of the indeterminacy thesis found in Word and Object as well as the accompanying argument which (mistakenly) received much attention as the main argument for the thesis. Quine's later arguments will then be put forth in an effort to both clarify the thesis and locate the strongest argument in support of it.

In Chapter 2 of Word and Object, Quine reveals his project, to "consider how much of language can be made sense of in terms of its stimulus conditions, and what scope this leaves for empirically unconditioned variation in one's scheme." 8 In other words, Quine wants to see just how much sense can be made of meanings (or sameness of meaning) within the limited realm of empirically conditioned (caused) stimulus responses, and if any sense can be made of meanings existing beyond this scope. He goes on to provide the following formulation of the indeterminacy thesis which is illustrated by attempting radical translation: 9

... manuals for translating one language into another can
be set up in divergent ways, all compatible with the
totality of speech dispositions, yet incompatible with one
another. In countless places they will diverge in giving, as

5 For the Sake of brevity I will provide only quotes which bear on the most immediate issues--explication and/or defense of the indeterminacy thesis, and assume the reader is otherwise familiar with Quine's writing.

6 Word and Object, section 15.

7 W.V. Quine, "On the Reasons for Indeterminacy of Translation," T/u fourmol of Philosophy, 1970, pp. 178-183, "Indeterminacy of Translation Again," Th" Journal of Philosophy, 1987, pp. 5-10, "Epistemology Naturalized," in Ontological Relativity and Other Essays, (New York: Columbia University Press, 1969), pp. 69-90.

Davidson, Donald, and Hintikka, Jaakko, eds., Words and Objections: Essmys on the Work of W.V. Quime, (Dordrecht, Holland: Reidel, 1969).

8 Word and Object, p. 26.

"Quine would later describe radical translation as a "thought experiment" designed to show that, "there is nothing to linguistic meaning . . . beyond what is to be gleaned from observable circumstances." "Indeterminacy of Translation Again," p. 5. 
their respective translations of a sentence of the one language, sentences of the other language which stand to each other in no sort of plausible equivalence, however, loose. The firmer the direct links of a sentence with nonverbal stimulation, of course, the less drastically its translation can diverge from one another from manual to manual.10

We learn that the thrust of the thesis lies in the possibility (or perhaps the inescapability) of generating divergent translation manuals which are constructed from, hence compatible with, the very sam evidence (verifiable speech dispositions). The degree of indeterminacy is a function of the sentence's (or term's) link to non-verbal stimulation; in Quine's terms, observation sentences will not suffer from much indeterminacy, while standing sentences suffer greatly.

The upshot of the thesis is that the common conception of meanings as unique and determinate entities is shattered; there are no such unique, fixed entities according to Quine, for their identity conditions cannot be established. That is, the very same behavior is evidence of more than one meaning; the evidence does not suffice to fix the conditions under which a particular meaning is said to be present or expressed. Of course, if there are no such fixed meanings, then it will make no sense to speak of sameness of meaning either, for there are no objective meanings for terms or sentences to share.

The remainder of Chapter 2 is primarily devoted to drawing out the indeterminacy thesis by utilizing radical translation. It is here that Quine provides his first argument for the indeterminacy thesis. The argument relies upon analytical hypotheses, 11 as well as what Quine later refers (t) as the inscrutability of reference. Quine writes:

... stimulus meaning was incapable of deciding among 'rabbit,' 'rabbit stage,' and various other terms as translations of 'gavagai' . . . [two different] analytical hypotheses may be presumed possible. Both could doubtless be accommodated by compensatory variations in analytical hypotheses concerning other locutions, so as to conform equally to all independently discoverable translations of while sentences and indeed all speech of all speakers concerned. And yet countless native sentences admitting no independent check ... may be expected to

10) Word and Object, p. 27.

11 Basically, these hypotheses equate (hypothetically) an English term or phrase with a Jungle term or phrase. Analytical hypotheses are suppessed to enable the translator to progress, albeit tentatively, with her project of radical translation. 
receive radically unlike and incompatible English renderings under the two systems. 12

It seems the argument here is intended to show that the epistemic evidence is such that it will equally support analytical hypotheses which translate the term 'gavagai' into quite different (or perhaps even contradictory) English terms or phrases (e.g. 'rabbit stage,' 'undetached rabbit part'). ${ }^{13}$ Moreover, the argument goes, the indeterminacy at the level of terms will promote indeterminacy on the level of sentences as well.

The obvious weakness in this argument is the vagueness surrounding the relationship between inscrutability of reference (which applies only to terms) and the indeterminacy of translation (which applies only to sentences). ${ }^{14}$ Quine later makes it quite clear that while inscrutability of reference may entail indeterminacy, this is not always the case. ${ }^{15}$ He states that;

[t]he gavagai example had only [an] indirect bearing on indeterminacy of translation of sentences: one could imagine with some plausibility [that inscrutability of terms will carry with it indeterminacy of sentences] . . . this whole effort was aimed not at a proof but at helping the reader to reconcile the indeterminacy of translation imaginatively with the concrete reality of radical translation. The argument for the indeterminacy is another thing, as seen earlier in this paper. ${ }^{16}$

Thus Quine admits that the foregoing argument was not intended to serve primarily as an argument, but rather as a device to facilitate understanding the thesis; instead he endorses another argument presented earlier in "On

12 Word an Object, pp. 71-72.

13 And to make the point of the thesis more dramatically, the evidence will never support just one unique translation, it will always leave translation indeterminate.

14 See, "Indeterminacy of Translation Again," pp. 8-9.

15 "A clear example [of inscrutability of reference] was seen in connection with the Japanese classifiers [Ontologicul Relutivily and Other Essinys, pp. 35ff.] This example makes it pretty clear, moreover, that the inscrutability of terms need not always bring indeterminacy of sentence translation in its train." "On the Reasons for Indeterminacy of Translation," p. 182. Here Quine also) comes to refer to this method of arguing from inscrutability to indeterminacy as "pressing from below," p. 183.

16 lbid., p. 182, and 178, where Quine writes, "My gavagai example has figured too centrally in discussions of the indeterminacy of translation. Readers [mistakenly] see the example as the ground of the doctrine." 
the Reasons for Indeterminacy of Translation." Let us consider this second argument.

While the previous argument was coined "pressing from below," the current argument came to be known as "pressing from above," the Quine considers it to be "very different, broader and deeper" than the former argument. 17 This method of arguing for the indeterminacy thesis relies upon the undetermination of physical science. Yet Quine goes out of his way to make it clear that indeterminacy is not merely a special case of underdetermination of physical theory; rather, indeterminacy is a point in addition to undetermination. ${ }^{18}$ Quine maintains that there is only a limitud parallel between the underdetermination and indeterminacy, and writes:

Thus, adopt for now my fully realistic attitude ... falling in with the current theory of the world despite knowing that it is in principle methodologically under-determined. Consider, from this realistic point of view, the totality of truths, known and unknown, observable and unobservable, past and future. The point about indeterminacy of translation is that zuithstands even all this Iruth, where indeterminacy of translation applies there is no real question of right choice; there is no fact of the matter even to wilhin the acknowledged underdetermination of a theory of nature. 19

So by appreciating both the underdetermination of physical theory and the fact that such theory is (to use Quine's words) the ultimate parameter for any attempts at radical translation, we come to realize that indeterminacy is suffered over and above underdetermination.

Quine emphasizes that there is a point at which deciding between competing translations, that is, deciding whether to attribute translation $A$ or translation $B$ to the native becomes, "a question whose very significance [he] would put in doubt." 20 We are now at the crux of the argument. Questions of meaning which seek a unique answer are misguided according to Quine, for there is, strictly speaking, nothing to be right or wrong about. If the empirical evidence does not favor one translation over another, then the two are equally plausible translations, even if they diverge radically in their assignments. There is nothing to which we may appeal beyond the empirical evidence. Moreover, this should not be understond as an epistemological point, but an ontological one. It is not a question of having enough information or evidence upon which to base our decision in.

17 lbid., p. 178.

Is Ibid., p. 180, see also the reply to Chomsky in Words and Objiclions, pp. 302-304.

19) Words and Oljections, p. 303, first emphasis is mine.

21) "On the Reasons for Indeterminacy of Translation," p. 181. 
translation, but rather that even once all the facts are in, there is simply nothing to be right or wrong about.

Let us consider a third and final argument for the indeterminacy thesis. This argument is located in "Epistemology Naturalized" and is generated out of combining Quine's verificationism and holism. Edward Becker provides a thorough synopsis of the argument he believes Quine to currently favor:

... a sentence is supposed to be synonymous with its translation. If following Peirce we adopt the verificationist theory of meaning, synonymy, sameness of meaning, will be sameness of confirming and disconfirming experiences. But it, following Duhem, we adopt a holistic view of confirmation, it will turn out that individual theoretical sentences do not have their own confirming and disconfirming experiences. Consequently, the notion of synonymy will not make sense in application to such sentences and it therefore will not make sense, a fortiori, to speak of a uniquely correct translation of such sentences. In this sense, their translation will be indeterminate. 21

Thus, it appears that indeterminacy is the natural outcome of adopting both a verificationist theory of meaning and a holistic view of confirmation.

Having somewhat briefly surveyed Quine's indeterminacy thesis and the supporting arguments, let us turn to Katz's proposed refutation of the thesis.

\section{Katz's Refutation of the Indeterminacy Thesis}

Katz's argument against the indeterminacy thesis garners plausibility from three primary assumptions: (i) that Quine's argument for the thesis is based or depends upon the inscrutability of reference, such as witnessed in the 'gavagai' example, (ii) that the distinction between actual translation and radical translation is a significant one, and (iii) that linguistically neutral meanings are the 'missing link' which, when resurrected, leave one free to pursue the parallel between translation and science (hence leave translation no worse off than a case of under-determination). 22 I suggest that these assumptions are mistaken, and it is in virtue of being so mistaken that Katz's argument fails as a refutation of Quine's thesis. I will now consider these assumptions in turn, explaining why I believe them to be erroneous.

21 Becker, Edward. Unpublished manuscript Chapter 3, section 3.

22 Katz provides his reconstruction of Quine's argument on pp. 237-238, and first refers tot he argument in "Two dogmas" as the 'missing' argument on $p$. 238. 
Assumption (i)

We recall from section 1 above that it is not necessarily the case that every instance of inscrutability of reference brings with it indeterminacy of translation. Moreover, we saw that Quine himself has all but abandoned "pressing from below," and has gone on to endorse two more powerful arguments which do not rely in any way upon inscrutability. This being the case, the indeterminacy thesis must be understood as independent of arguments generated from the inscrutability of terms. Yet Katz reconstructs Quine's argument as one which hinges upon the indeterminacy present in reference. Katz writes:

\begin{abstract}
Although nothing near a proof ["pressing from below"], the argument exhibits an unbreakable symmetry among the evidential considerations that can be adduced to justify various translations. . . [t]he reason is that the ostensive acts of the field linguist and the informant cannot refer to a rabbit without referring to a rabbit stage or undetached rabbit part, nor any of these without the others, and radical translation contains nothing that enables the linguist to impose controls on hypotheses which enable them to choose between extensionally equivalent translation options. thus, the argument leave us with not grounds on which to resist its conclusion. 23
\end{abstract}

Thus Katz believes the reason that various translations can be justified given the evidence is twofold: reference is inscrutable, and radial translation contains nothing that would enable the translator to impose controls on the hypotheses, hence reach a decisively better or correct translation. The latter point will be discussed shortly in terms of assumption (iii). It should now be obvious that linking inscrutability in some necessary fashion with indeterminacy is a mistake. Katz has neglected to address Quine's later, more powerful arguments for indeterminacy.

Assumption (ii)

Katz is convinced that actual translation is significantly different than radical translation, and that this difference effects Quine's argument, Katz writes:

I concede that there is no doubt about Quine's conclusion if restricted to radical translation. But it is not clear how actual translation, to which Quine's conclusion must apply if indeterminacy is to matter philosophically, is related to

23 "The Refutation of Incteterminacy," p. 231. 
radical translation. Thus ... there can be doubts about the step in Quine's argument from his account of radical translation to his conclusion as applied to actual translation ... [t] the acceptability of an identification depends on whether actual translation is in all relevant respects like radical translation. ${ }^{24}$

Katz goes on to say that surely one relevant respect in which radical and actual translation must be similar is the absence of "independent controls" which would allow the translator to get past the evidential symmetry, thus eliminating indeterminacy of translation as well.

There are two reasons why Katz's point is misguided. First, Katz overstates the importance of the distinction between radical and actual translation. Katz accepts Quine's behaviorist approach as, "a behaviorism one can live with."25 This behaviorism is really all that need be assumed to produce the philosophically interesting conclusion. Radical translation is simply an experiment whereby behavior (which Katz agrees with Quine is) relevant to meaning is highlighted. In "indeterminacy of Translation Again," Quine portrays radical translation as thought experiment in which, "our only data are native utterances and their concurrent observable circumstances." 26 Thus, for Katz to attack the distinction between radical and actual translation, it seems he would also need to criticize Quine's apparently innocuous behaviorist approach.

The second reason Katz's point concerning the distinction between radical and actual translation is mistaken pertains to the existence of independent controls which would facilitate deciding between competing analytical hypotheses. Katz claims that a relevant distinction between radical and actual translation which Quine neglects is that of the existence of such independent controls in actual, but not in radical, translation; "it is the absence of such controls which causes evidential symmetry and indeterminacy." Furthermore, Katz believes the existence of such controls is in turn wholly dependent upon the existence of linguistically neutral meanings. Thus, Katz reasons, if we can establish the existence of linguistically neutral meanings, we will then have grounds for imposing independent controls upon competing analytical hypothesis, triumphantly break the evidential symmetry, and topple the indeterminacy thesis. The path will then be clear to pursue the parallel between translation and science (that is, translation will simply be another case of underdetermination).

This relates to assumption (iii) above which I initially mentioned as one of Katz's crucial assumptions. But first let us dispense with the question of whether or not Katz is correct in necessarily linking the existence of independent controls to the existence of linguistically neutral meanings.

24 Ibid., pp. 232-233, his emphasis.

25 lbid., p. 231.

26 Indeterminacy of Translation Again," p. 5. 
Surely he is not correct. For one can obviously impose any independent controls one wishes, without implying or requiring the existence of any particular entities; the controls simply serve as an objective criterion according to which one may designate particular terms or sentences as, say synonymous. This does not, however, mean that there must be some objective entities according to which these controls coincide. In other words, establishment of independent controls is a separate matter from establishment of the identify conditions for objectively existing meanings. Thus, the necessary connection between independent controls and linguistically neutral meanings reflects a highly dubious step in Katz's argument. 27

\section{Assumption (iii)}

Finally, after seeing that Katz has mistakenly backed the question of the existence of independent controls up to that existence of linguistically neutral meanings, we reach assumption (iii): that linguistically neutral meanings represent the 'missing link' in Quine's argument for the indeterminacy thesis and, if resurrected, will insure the existence of independent controls, which, in turn, enable us to break the evidential symmetry, and overcome indeterminacy intranslation. But this premise is absurd. For in addition to the point just made that linguistically natural meanings are not necessarily linked with independent controls, to begin the refutation with a quest for linguistically neutral meanings reflects Katz's attribution of a viciously circular (not to mention inaccurate) argument to Quine.

Katz would have us look back to "Two Dogmas Of Empiricism" 28 to locate the foundation of Quine's argument for indeterminacy. If, as Katz insists, Quine had annihilated meanings in "Two Dogmas of Empiricism," and if this argument was later intended to serve as the basis of the argument for indeterminacy, the Quine is involved in a blatant and inescapable circle of reasoning. Here, quite simply, is why: the majority goal or conseifuence of the indeterminacy thesis is proclaimed by Quine to be the, "challenge of an ill-conceived notion within traditional semantics, namely, sameness of meaning." Clearly, if Quine's objective is to undermine the traditional, uncritical notions of meaning and sameness of meaning, then it would be on pain of circularity that we would attribute the annihilation of meanings as the starting point of Quine's argument for indeterminacy.

I suggest, against Katz, that "Two Dogmas" was merely an earlier attempt (if from a different angle), to question the sense of the traditional notions of meaning and sameness of meaning. Thus, we should view both

$27 \mathrm{Katz}$ is thus incorrect in stating that the, "reason for thinking independent controls do not exist in translation is only as good as the reason Quine has for saying that there are no linguistically neutral meanings," p. 234.

${ }^{28}$ W. V. Quine, From a Logical Point of View (Cambridge: Harvard, 1953). 
"Two Dogmas" and the subsequent indeterminacy arguments as attempts at the very same goal, namely, "to change the prevalent attitudes toward meaning, idea, proposition. And in the main the sad fact is . . . [this] escapes recognition precisely because of the uncritical persistence of old notions of meaning, idea, proposition." 29 A sad fact indeed. 